\title{
Measurement of human exposure to biologically relevant fractions of inhaled aerosols
}

\author{
John W Cherrie, Robert J Aitken
}

\begin{abstract}
Aerosol sampling has evolved with changes in technology and our understanding of the importance of assessing the biologically relevant fractions of the total aerosol. During the past decade there has been international agreement on the definitions for the inhalable, thoracic, and respirable fractions and instruments have been developed to collect samples according to these conventions. These measurement techniques are now well established in the workplace and are increasingly being applied to assessments of nonoccupational exposure, as the practical difficulties in obtaining samples over 24 hours have been solved. It is argued that multiple aerosol size fractions should be measured for hazardous substancessuch as inorganic lead-in which inhaled material may either be absorbed in the alveoli (respirable) or cleared from the ciliated airways in the lung to the gut and then absorbed (thoracic and extrathoracic). Such measurements should improve the evaluation of the risk for inhaled lead, particularly for non-occupational exposure of children. Also, passive aerosol sampling techniques may enable measurements of non-occupational exposure to be made over several weeks and this would also help improve the reliability of the risk evaluation for aerosol toxicants with a long biological half life. However, further development of these techniques is required if they are to be practicable and biologically relevant.

(Occup Environ Med 1999;56:747-752)
\end{abstract}

Keywords: exposure; thoracic; lead

It has long been recognised that the presence of particles in the air may adversely affect the health of exposed people. However, it is only in the past 100 years that serious attempts have been made to measure the size, shape, and concentration of airborne particles and to then relate these measures to human health. Inevitably, almost all of the early work was involved with measurement of aerosols in occupational settings such as mining, as it was in these situations that effects on health were most obvious.

Most of the early aerosol monitoring devices were designed to be placed in one place while the sample was collected. However, the advent of small and light battery operated pumps in the 1960s meant that personal exposure measurements became possible. Early personal samplers comprised simple open faced filter holders that were thought to collect "total dust". More complex devices, such as cyclones designed to collect only the finer fractions of airborne dust, became available only later. However, these improvements in technology enabled the links to be identified between occupational exposure to a range of aerosols and lung disease.

Developments in monitoring of exposure to aerosols have generally been focused towards sampling of occupational exposure, although the principles are equally applicable to nonoccupational exposures. In the past, limitations on analytical sensitivity have dictated that environmental sampling of aerosols should be undertaken with high volume fixed location monitoring equipment. This is no longer the case and personal exposure measurements in non-occupational situations should help improve risk assessments.

In this paper we briefly review some of the recent developments in sampling methods used to assess human exposure to aerosols, and in particular, we look at issues of transferring experience from workplace sampling to nonoccupational exposure monitoring. We use nonoccupational exposure to inorganic lead aerosol as an example of the issues involved in developing an appropriate measurement strategy.

Early developments in aerosol sampling Up to the mid-1920s the principal method of sampling aerosols was the sugar tube, later described by Walton. ${ }^{1}$ This device comprised a $32 \mathrm{~mm}$ diameter tube filled to a depth of about $100 \mathrm{~mm}$ with 10-20 mesh sugar granules. Air was drawn through the granules by a hand operated pump and the collected dust was analysed by dissolving the sugar, and filtering the residue, which was then weighed to provide an estimate of the airborne mass. As well as being difficult to use, this method was criticised because reductions in the measured dust concentration in mines and other dusty environments were not matched by corresponding falls in dust related diseases.

More complex instruments soon became available. One such device was the konimiter, which was developed in 1916 by Kotze. ${ }^{2}$ In this device, a small sample of air was drawn through a jet nozzle and directed towards a plate covered with petroleum jelly where the particles deposited by impaction. The plate was removed and the particles counted with a microscope. The operating principles of this device recognised that both the particle numbers and the particle size could be important determinants of risk. However, it was only able to collect a sample over a very short duration (about 1 second). 
By the mid-1930s there were several alternatives to the konimiter, the most important being the thermal precipitator. ${ }^{3}$ This overcame the limitations of the konimiter to collect very small particles $(<1 \mu \mathrm{m})$ and samples could be collected over much longer periods. The thermal precipitator had an inlet leading to a narrow channel where there was a heated wire oriented perpendicular to the direction of flow. As the contaminated air passed the wire, thermal gradients caused the particles to be deflected towards the walls of the sampler where two glass disks were located. After sampling, the glass disks were removed and the particles counted as with the konimiter. The thermal precipitator was a heavy bulky instrument that could only be used for fixed location measurements.

Partly because of ease and reliability of analysis, gravimetric sampling with filters to collect the dust from the air largely superseded particle counting methods. An early example of such an instrument is the Mine Research Establishment (MRE) type 113A gravimetric sampler ${ }^{4}$ which has been used in the mining industry in the United Kingdom to collect the British Medical Research Council (BMRC) respirable dust fraction. ${ }^{5}$ The respirable fraction was defined qualitatively as the fraction of the airborne dust that approximates to that which would reach and deposit in the alveoli, reflecting the belief that these particles were of greater importance in causing occupational lung disease, especially pneumoconiosis. With this device a close relation was established between the prevalence of pneumoconiosis and the mass concentration of respirable coal mine dust, ${ }^{6}$ whereas earlier attempts to correlate disease with the number of particles of dust had been less successful. ${ }^{7}$ The development of the BMRC respirable fraction was the first of what have become known as the "biologically relevant" aerosol size fractions that now form the basis of modern sampling methods.

\section{Sampling of biologically relevant and other aerosol size fractions}

Over the past 10 years substantial progress has been made towards standardising the sampling of aerosols to ensure that the fractions sampled are biologically relevant. In 1993 a new European standard for the definition of size fractions for measuring airborne particles was approved by the European Committee for Standardisation. ${ }^{8}$ This standard defines three sampling conventions: inhalable fraction (the mass fraction of airborne particles which is inhaled into the nose or mouth); thoracic fraction (the mass fraction of inhaled particles penetrating beyond the larynx) and respirable fraction (the mass fraction of inhaled particles penetrating to the unciliated airways). These three criteria are shown in figure 1. The same definitions, for workplace atmospheres, have also been adopted by the International Standards Organisation ${ }^{9}$ and The American Conference of Governmental Industrial Hygienists (ACGIH). ${ }^{10}$

These conventions recognise that only part of the total aerosol is inhaled into the nose or mouth (the inhalable fraction). This concept was based on measurements of the aspiration efficiency of life sized human models reported by Ogden and his co-workers ${ }^{11}{ }^{12}$ and subsequently by Vincent and his colleagues. ${ }^{13-15}$ For large particles $(>50 \mu \mathrm{m}$, aerodynamic diameter) only about $50 \%$ of the total aerosol enters the respiratory tract according to the inhalable criteria, and this increases to almost $100 \%$ for small ( $\sim \mu$ m diameter) particles. The thoracic and respirable conventions are subfractions of the inhalable aerosol and have their basis in a wide range of human volunteer deposition and clearance studies. ${ }^{16}$ However, the final forms of the definitions were a compromise in the case of the respirable convention between the BMRC curve and the previous ACGIH definition. ${ }^{17}$

The development of size conventions for measurement of ambient aerosol has been through a separate process to that of the workplace definitions and has been driven primarily by the deliberations of the United States Environmental Protection Agency. Greater emphasis in these considerations has been placed on the size distribution of aerosol in the ambient air but potential penetration into the human respiratory tract was also considered. The two commonly used environmental sampling conventions are $\mathrm{PM}_{10}$ and $\mathrm{PM}_{2.5}$ both of which are curves falling from unity to zero with, in the case of $\mathrm{PM}_{10}$, a $50 \%(0.5)$ value at $10 \mu \mathrm{m}$ and, for $\mathrm{PM}_{2.5}$ a 0.5 value at $2.5 \mu \mathrm{m}$. Although not specifically designed to be so, the thoracic convention is almost identical to the $\mathrm{PM}_{10}$ size selection curve. There is, however, a significant difference between the respirable convention and the $\mathrm{PM}_{2.5}$ curve.

It is now considered that personal samplers are much more effective tools for assessing exposure to aerosols in the workplace than static samplers. The types and performance of these devices have been described by other authors. ${ }^{18}$ In the main, these samplers have been targeted to meet either the inhalable convention-for example, the Institute of Occupational Medicine personal inhalable sampler $^{19}$ - or the respirable convention-for example, the Higgins cyclone. ${ }^{20}$ As yet there are no commercial samplers for the thoracic convention although several research devices have or are being developed. ${ }^{21}{ }^{22}$ The clear rationale is that sampling personal exposure provides a better evaluation of potential risks, as it has also been shown that personal

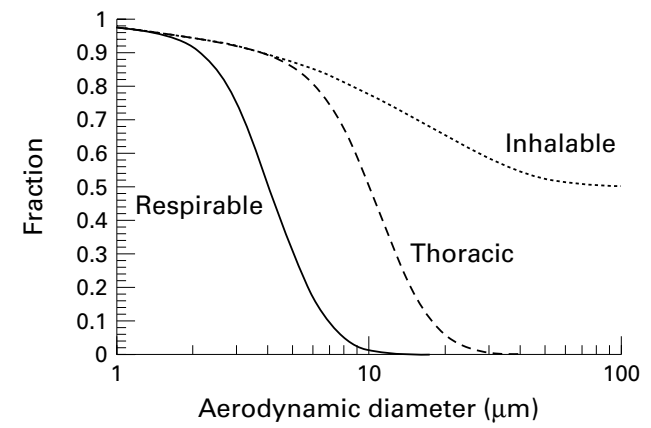

Figure 1 Inhalable, thoracic, and respirable sampling criteria. 
exposures are almost invariably higher than those found with simultaneously operating fixed location monitors. ${ }^{23}$

\section{Measurement of exposure in non-occupational groups}

Procedures for monitoring personal exposure to airborne particles in non-occupational situations have been adapted from those used in the workplace. The equipment generally comprises a small battery operated pump connected to a size selective sampling head containing a filter paper or some other particle collection system. The main problems associated with these techniques arise because of the low airborne mass concentrations in nonoccupational situations and the necessity to sample over relatively long periods, typically 24 hours or more. As the person should wear the pump throughout the sampling period, except during sleep, it must be lightweight and quiet and this limits the maximum airflow. As a consequence, the minimum detectable mass concentration is generally about $10 \mu \mathrm{g} / \mathrm{m}^{3}$ over 24 hours. ${ }^{24}$ The detection limit for components of the aerosol, such as lead, will be determined by the sensitivity of the analytical techniques used, but could be much less than for gravimetric assessments, perhaps about $10 \mathrm{ng} / \mathrm{m}^{3}$ for lead. ${ }^{25}$

During personal sampling the entry into the sampling head is normally located close to the person's nose and mouth, typically on the outside of their clothing on a lapel to give an estimate of the concentration of particles inhaled. During the hours of sleep the sampler is generally placed in the individual's bedroom. To ensure that the noise of the sampler does not disturb the person during sleep some investigators have devised sound insulated boxes to muffle the pump noise. ${ }^{24}$ These systems may also be used at other times when the noise would be distracting - for example, while watching television or reading. It is necessary to replace the pump battery about every 8 hours or to use mains electricity overnight.

There are several sampling heads that have been used to measure personal exposure to airborne particles. Measurements made more than 15 years ago may have been made as total suspended particulate (TSP), although this is a misnomer and these instruments generally had an undefined sampling characteristic which would have included some, but not all, of the larger particles suspended in the air. More recently measurements have been made with the size selective sampling criteria described earlier. Even with such systems there is still the possibility that wearing a noisy pump may alter the person's behaviour and bias the measured exposure level. ${ }^{2627}$

There are a few studies, mostly carried out over the past 10 years, which provide reliable measurements of personal exposure to airborne particles. These studies, which have been carried out in North America and more recently in Europe, do not generally measure the metal content of the aerosol. Most of the data refer to urban or suburban populations, generally excluding workers in occupations where there is known exposure to aerosols, and most measurements have been made over 24 hours. In total, there are about 2000 published measurements from various subgroups within society and only about 1200 measurements made in relation to currently accepted biologically relevant sampling conventions - such as thoracic aerosol (table).

In these studies the mean thoracic aerosol concentration ranged from $42 \mu \mathrm{g} / \mathrm{m}^{3}$ for the study of Seaton et $a^{28}$ of elderly volunteers in Belfast to $105 \mu \mathrm{g} / \mathrm{m}^{3}$ for children in The Netherlands. ${ }^{29}$ Most of these measurements were made over 24 hour periods and where this was not the case (Birmingham, UK) the data have been adjusted to estimate 24 hour exposure by using the average fixed point monitoring data to represent the unmeasured time. Other studies have shown that during the night indoor, outdoor, and personal concentrations agree quite well and so this adjustment seems justifiable. ${ }^{30}$ The maximum individual thoracic concentration was $971 \mu \mathrm{g} / \mathrm{m}^{3}$ (24 hour average), measured by Loiy et al in Phillipsburg, $\mathrm{NJ} \cdot{ }^{31}$

Sampling human exposure to lead aerosol Sampling of lead aerosol in occupational situations is one of the few cases where current sampling practice does not require biologically relevant size selective sampling, although the method has been standardised. There is no agreement about how lead should be sampled outside the workplace.

There is also very little information about the size range of lead aerosols in ambient air in the United Kingdom. From the Quality of Urban Air Review Group (QUARG) report on airborne particulate matter in the United Kingdom ${ }^{32}$ about $90 \%$ of the lead aerosol is in the fine fraction (aerodynamic diameter $<2.5$

Measurements of thoracic aerosol exposure in various groups of non-occupationally exposed subjects over 24 hours

\begin{tabular}{|c|c|c|c|c|c|c|}
\hline Study & Location & Study group & $\begin{array}{l}\text { Subjects } \\
(n)\end{array}$ & $\begin{array}{l}\text { Measurements } \\
(n)\end{array}$ & $\begin{array}{l}\text { Geometric } \\
\text { mean exposure } \\
\left(\mu g / m^{3}\right)\end{array}$ & $\begin{array}{l}\text { Maximum } \\
\text { exposure } \\
\left(\mu g / m^{3}\right)\end{array}$ \\
\hline Lioy $e t a l^{\beta 1}$ & Phillipsburg, USA & Non-smoking volunteers & 14 & 189 & 66 & 971 \\
\hline Pellizzari et $a l^{30}$ & Riverside, USA & Representative selection of non-smokers & 178 & 171 & $98^{\star}$ & $285 \dagger$ \\
\hline Janssen et al ${ }^{9}$ & Amsterdam and Wageningen, Netherlands & Children aged $10-12$ y & 45 & 301 & $105 \ddagger$ & 195 \\
\hline Mark et $a l^{42}$ & Central Birmingham, UK & Volunteers, smokers, and non-smokers & 22 & 127 & $70 \S$ & 260 \\
\hline Mark et $a l^{42}$ & East Birmingham, UK & Volunteers, smokers, and non-smokers & 15 & 178 & $47 \S$ & 120 \\
\hline Janssen et al $l^{43}$ & Amsterdam, Netherlands & Non-smoking volunteers aged $50-70 \mathrm{y}$ & 37 & 262 & $59 \neq$ & 113 \\
\hline Seaton et $a l^{8}$ & Edinburgh, UK & Volunteers aged $>60 \mathrm{y}$ & 57 & 57 & 57 & 418 \\
\hline Seaton $e t a l^{28}$ & Belfast, UK & Volunteers aged $>60 \mathrm{y}$ & 52 & 54 & 42 & 288 \\
\hline
\end{tabular}

^Population weighted median exposure rather than geometric mean

†Maximum is the estimated 98 th percentile.

$\ddagger$ Geometric mean estimated from arithmetic mean (SD).

SEstimated geometric mean and maximum, see text for explanation. 
$\mu \mathrm{m})$, probably associated with particles $<1 \mu \mathrm{m}$ in diameter. ${ }^{33}$ It is possible that there may be greater concentrations of lead in the coarse fraction when lead contaminated dust, which is attached to other settled dust in the environment, is then resuspended. Because of the bonding of particles to surfaces, most dust which is resuspended will have a diameter $>1-5 \mu \mathrm{m} .{ }^{34}$ Measurements of lead in household dust suggest that there may be substantial amounts of lead associated with larger particles. $^{35}$

When considering which size fraction or fractions are most appropriate for sampling human exposure to lead aerosol, we should consider the fate of the different size particles. Ideally, the measurement system should be capable of collecting the inhalable and respirable fractions. For lead particles which deposit in the alveolar region there is almost $100 \%$ absorption. However, only about $10 \%$ of the lead that is swallowed by adults is absorbed into the blood, although the proportion absorbed by children is higher, perhaps $30 \%$ to $40 \%{ }^{36}$ The inhalable fraction less the respirable fraction represents that part of the aerosol which could potentially be absorbed through the gut and the respirable fraction that part which would be absorbed through the lung. These two measurements could then be combined as a weighted sum, based on the approximate efficiency of intake from each route of exposure. Alternatively, such data would provide reliable input data for developing the physiologically based pharmacokinetic models available for lead. ${ }^{37}$

The United Kingdom Expert Panel on Air Quality Standards has recommended that there should be a standard of $0.25 \mu \mathrm{g} / \mathrm{m}^{3}$ for lead, averaged over a year. ${ }^{38}$ This standard is intended to protect the intellectual development of young people from inhaled lead aerosol. Unfortunately, the standard does not precisely specify the sampling procedure to be used and so it is unclear what size fraction it includes. For the fixed point measurements made by the United Kingdom Department of Environment, Transport, and the Regions this is probably unimportant because their samplers are generally located far from sources of coarse particles and most available samplers should give comparable results. However, differences in instrumentation would be much more important if the air quality standard were applied to personal exposure monitoring.

There is very little information on exposure to lead aerosol in the general population and the data that do exist have been obtained with samplers that do not conform to the agreed size selective sampling criteria. $^{25}$ Nevertheless, these data suggest that week long average lead exposures in Swedish adults range between 0.04 and $0.09 \mu \mathrm{g} / \mathrm{m}^{3}$, as total dust. In the United Kingdom measurements of lead in the general atmosphere suggest that it makes up about $0.2 \%$ by mass of the thoracic $\left(\mathrm{PM}_{10}\right)$ aerosol. Applying this factor to the data shown in the table, assuming most of the variability in measured values is associated with the people rather than the day of sampling, suggests that the average exposure level would be about $0.1 \mu \mathrm{g} / \mathrm{m}^{3}$ and only about $5 \%$ of adults might exceed the United Kingdom air quality standard for lead. However, with the data from children there might be about half who could exceed the standard and it is this group who are especially at risk from lead exposure. Also, children living in homes where there was excessive lead contamination from flaking paint or from localised industrial sources might have personal exposures well above the standard.

\section{Passive aerosol samplers}

The appropriate averaging time for measurement should be dictated by the biological half life of the pollutant in the body with short term variations in exposure level being less relevant. ${ }^{39}$ In the case of lead the biological half life is about 35 days $^{33}$ and this would be an appropriate averaging period. Clearly, measurement of personal lead exposure with conventional sampling strategies over such a long period is impracticable and the available data do not easily allow extrapolation to estimate such exposure. It might be possible to sample 24 hour average exposure on several occasions throughout a 5 week period and this would approximate to the long term exposure level, but this would still be costly and inconvenient for the subjects.

Sampling of gases and vapours has been revolutionised by the introduction of small lightweight samplers that do not have a pump but rely on the principle of diffusion to collect the material. Over the past 10 years there have been attempts to develop a similar approach for aerosols. The most promising of these, shown in figure 2, comprises a $25 \mathrm{~mm}$ diameter electret material held within an electrically conducting holder. ${ }^{40}$ Electrets are polymers that have been treated to induce a permanent electrical charge within their structure, with the charge at the surface of the polymer as high as $1000 \mathrm{~V}$. The sampler has a $1 \mathrm{~cm}$ gap between the surface of the electret and the front plate of the sampler and any particles that pass into this volume will drift towards the electret with a velocity proportional to their electrical mobility. The rate of collection of particles is relatively independent of their size, charge

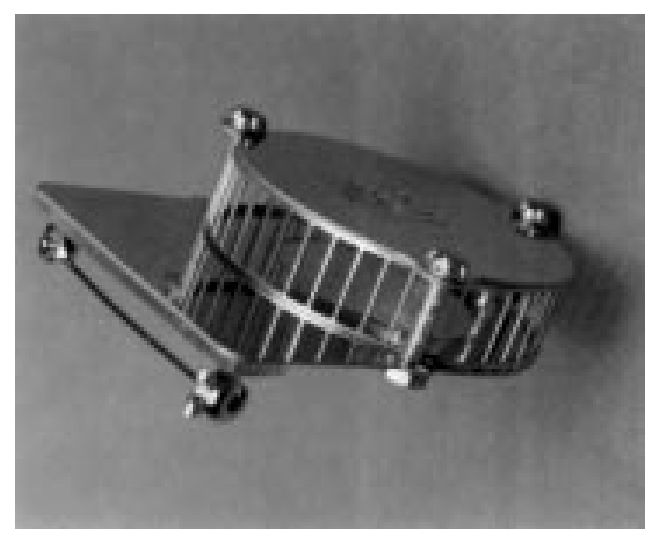

Figure 2 (A) Passive aerosol sampler developed by the United Kingdom Health and Safety Laboratory. 
distribution, or velocity, but this is highly dependent on the electrical mobility and this depends on the chemical composition of the aerosol. ${ }^{41}$

The sampler is small, lightweight (about 15 $\mathrm{g}$ ), and not intrusive. It may be worn for extended periods and requires almost no operator maintenance over that period. Therefore it would be an excellent device to assess aerosol exposure of non-occupational groups and could be used to measure exposure to lead, although there are several limitations that make its use problematic. The rate of collection by the device is very low. In a study which is currently underway to assess the use of the device in assessing urban domestic aerosols, collected masses over 2 week periods were of the order of $50 \mu \mathrm{g}$. Also, in its present form the sampler is not size selective. In principle at least, it collects all sizes with equal efficiency, although the range of sizes over which this holds has not been measured. Finally, as already described, the collection rate is dependent on the electrical mobility, and hence composition of the particles. It is therefore necessary to calibrate the collection rate for different types of aerosol by comparing the passive sampler with a conventional pumped sampler in each case. This may lead to the requirement for material specific designs, coupled with appropriate analytical methods to be produced. Further work is necessary if these limitations are to be overcome.

\section{Conclusions}

There have been many developments in the sampling of aerosols over the past decade. In this paper we have highlighted the important attempts to standardise the fraction of the aerosol that is sampled so that it roughly corresponds to the fraction penetrating to sections of the respiratory tract. There is now, for the first time, agreement between all those involved in sampling workplace aerosols to use the same definitions so that measurements of respirable aerosol made in the United States, Europe, and other parts of the world are comparable. It would be advantageous for those involved with sampling non-occupational aerosols to ensure that their measurements are obtained to the same standard.

A consequence of moving to biologically relevant size fractions is the desirability of obtaining measurements of multiple size fractions for some aerosols. We have used the example of lead, where measurement of the inhalable and respirable aerosol might be appropriate. Use of this type of approach would certainly allow a more precise estimate of the biologically relevant exposure to be made and would enable more accurate estimates of risk from inhaled aerosol. It is more important to select an appropriate sampling system for personal exposure measurements than for fixed point monitoring because of the likelihood of resuspended dust playing a greater part and this should be carefully considered when sampling aerosols outside the workplace.
Finally, selecting an appropriate averaging time provides a particular challenge for all hazardous aerosols that have a chronic effect on health. Here there is an advantage in having sample averaging times as long as possible, consistent with the biological half life of the pollutant, so as to reduce the variability associated with short term samples. Continuous personal sampling for aerosols over several days or weeks is currently impracticable, but further development of passive samplers offers the possibility of adopting such strategies in the future. The key difficulty will be to develop systems which combine the need to obtain size selective samples with that of measuring over prolonged periods.

We are grateful to Dr Nigel Crawford and Mr Fintan Hurley at the Institute of Occupational Medicine for their helpful comments, and to Dr Lee Kenny at the Health and Safety Laboratory (Sheffield) for the photograph of the passive aerosol sampler.

1 Walton WH. The nature, hazards and assessment of occupational exposure to airborne asbestos dust: a review. Ann Occup Hyg 1982;25:117-247.

2 Kotze RN. Final report of the Miners' Phthisis Committee. Pretoria: GPSO, 1919.

3 Green HL, Watson HH. Physical methods for the estimation of dust hazard in industry. London: Medical Research Council, 1935. (Special Reports Series No 199.)

4 DunmoreJH, Hamilton RJ, Smith DSC. An instrument for the sampling of respirable dust for subsequent gravimetric assessment. Fournal of Scientific Instruments 1964;41:66972 .

5 Orenstein AJ, ed. Recommendations adopted by the Pneumoconiosis Conference. In: Proceedings of the Pneumoconiosis Conference, fohannesberg, 610-21. London: Churchill, 1959

6 Jacobsen M, Rae S, Walton WH, et al. The relationship between pneumoconiosis and dust exposure in British coal mines. In: Walton WH, ed. Inhaled particles III. Old Woking: Unwin, 1971:903-17.

7 Rogan JM, Attfield MD, Jacobsen M, et al. Role of dust in the working environment in the development of chronic bronchitis in British coalminers. Br f Ind Med 1967;30: 217-26.

8 British Standards Institute. Workplace atmospheres: size fraction definitions for measurements of airborne particles. London: BSI, 1993. (BS EN 481.)

9 International Standards Organisation (ISO). Air quality: particle size fraction definitions for heath-related sampling. Geneva: ISO, 1993. (ISO CD 7708.)

10 American Conference of Governmental Industrial Hygienists (ACGIH). Threshold limit values for chemical substances and physical agents. Cincinnati, Ohio: ACGIH, 1993.

11 Ogden TL, Birkett JL. The human head as a dust sampler. Walton WH, ed. Inhaled particles IV. Oxford: Pergamon, 1977.

12 Ogden TL, Birkett JL, Gibson H. Improvements to dust measuring techniques. Edinburgh: Institute of Occupational Medicine, 1977. Final report on CEC Contract 6253-22/ $8 / 016$.

13 Vincent JH, Armbruster L. On the quantitative inhalability of airborne dust. Ann Occup Hyg 1981;24:245-8.

4 Vincent JH, Mark D. Application of blunt sampler theory to the definition and measurement of inhalable dust. In: WH Walton, ed. Inhaled particles $V$. Pergamon Press, Oxford 1982:3-19.

15 Armbruster L Bruer H (1982). Investigations into defining inhalable dust. In: WH Walton, ed. Inhaled particles $V$. Oxford: Pergamon Press, 1982:21-32.

16 International Commission on Radiological Protection. International Commission on Radiological Protection.
Human respiratory tract model for radiological protection.

17 Soderholm SC. Proposed international conventions for particle size selective sampling. Ann Occup Hyg 1989;33:30120.

18 Vincent JH. Aerosol science for industrial hygienists. Oxford: Elseivier, 1995.

19 Mark D, Vincent JH. A new personal sampler for airborne total dust in workplaces. Ann Occup Hyg 1986;30:89-102.

20 Higgins RI, Dewell P. A gravimetric size-selective personal dust sampler. Davies EN, ed. Inhaled particles II. Oxford: Pergamon, 1967:575-86.

21 Aitken RJ, Vincent JH, Mark D. Application of porous foams as size selectors for biologically relevant samplers. Applied Occupational and Environmental Hygiene 1993;8: 363-9.

22 Kenny LC, Stancliffe JD, Crook B et al. Development and evaluation of a personal bioaerosol sampler. Fournal of Aerosol Science 1998;29:S497-8. 
23 Cherrie JW. The effect of room size and general ventilation on the relationship between near and far-field concentrations. Applied Occupational and Environmental Hygiene (in press).

24 Janssen N, Hoek G, Harssema H, et al. Personal sampling of airborne particles: method performance and data quality. Fournal of Exposure Analysis and Environmental Epidemiology 1998;8:37-49.

25 Vahter M, Berglung M, Lind B, et al. Personal monitoring of lead and cadmium exposure: a Swedish study with reference to methodological aspects. Scand $\mathcal{f}$ Work Environ Health 1991;17:65-74.

26 Cherrie JW, Lynch G, Bord BS, et al. Does the wearing of sampling pumps affect exposure?. Ann Occup Hyg 1993;38: 827-38.

27 Fugas M. Monitoring of personal exposures to air pollutants: subjects' experience. Achiv za Hygijenu Rada I Toxsikologiju 1995; 46:237-41.

28 Seaton A, Godden D, Cherrie J, et al. Air pollution and cardiovascular disease: an investigation of the relationship between particulate air pollution and blood coagulation factors. Final report to the Department of Health for project 121/6281. Final report to the Department of Health for

29 Janssen NAH, Hoek G, Harssema H, et al. Childhood exposure to $\mathrm{PM}_{10}$ : relation between personal, classroom, and outdoor concentrations. Occup Environ Med 1997;54:88894.

30 Pellizzari ED, Thomas KW, Clayton CA, et al. Particle total exposure assessment methodology (PTEAM): Riverside, California Pilot Study. Vol 1. Research Triangle Park: Environmental Protection Agency, 1993. (EPA/600/SR-93/ 050.)

31 Lioy PJ, Waldman JM, Buckley T, et al. The personal, indoor and outdoor concentrations of $\mathrm{PM}_{10}$ measured in an industrial community during winter. Atmospheric Environment 1990;24:57-66.
32 Harrison RM, Brimblecombe P, Derwent RG, et al. Airborne particulate matter in the United Kingdom. Third Report of the Quality of Urban Air Re

33 International Programme on Chemical Safety. Inorganic lead.Geneva: World Health Organisation, 1995. (Environmental health criteria 165 .

34 Thatcher TL, Layton DW. Deposition, resuspension and penetration of particles within a residence. Atmospheric Environment 1995;29:1487-97.

35 Wang EY, Willis RD, Buckley TJ, et al. The relationship between the dust lead concentration and the particle sizes of household dusts collected in Jersey City residences. Appl Occup Environ Hyg 1996;11:99-206.

36 Ziegler EE, Edwards BB, Jensen RL, et al. Absorption and retention of lead in infants. Pediatr Res 1978;12:29-34.

37 White PD, Van Leeuwen P, Davies BD, et al. The conceptual structure of the integrated exposure uptake biokinetic model for lead in children. Environ Health Perspect 1998;106(suppl 6):1513-30.

38 Expert Panel on Air Quality Standards, Lead. London: HMSO, 1998.

39 Rappaport S.M. Assessment of long-term exposures to toxic substances in air. Ann Occup Hyg 1991;35:61-121.

40 Brown RC, Wake D, Thorpe M, et al. A passive sampler for airborne dust using an electret. Fournal of Aerosol Science 1992;23:S623-6

41 Brown RC, Wake D, Thorpe $\mathrm{M}$, et al. Preliminary assessment of a device for passive sampling of airborne particulate. Ann Occup Hyg 1994;38:303-18.

42 Mark D, Upton SL, Lyons CP, et al. Personal exposure measurements of the general public to atmospheric particles. Ann Occup Hyg 1997;41 (suppl 1):700-6.

43 Janssen NAH, Hoek G, Brunekreef B, et al. Personal sampling of particles in adults: relation among personal, indoor and outdoor concentrations. Am $\mathcal{f}$ Epidemiol 1998;147:537-47.

\section{Vancouver style}

All manuscripts submitted to Occup Environ Med should conform to the uniform requirements for manuscripts submitted to biomedical journals (known as the Vancouver style.)

Occup Environ Med, together with many other international biomedical journals, has agreed to accept articles prepared in accordance with the Vancouver style. The style (described in full in the $f A M A[1]$ ) is intended to standardise requirements for authors, and is the same as in this issue.

References should be numbered consecutively in the order in which they are first mentioned in the text by Arabic numerals on the line in square brackets on each occasion the reference is cited (Manson[1] confirmed other reports[2][3][4][5]). In future references to papers submitted to Occup Environ Med should include: the names of all authors if there are three or less or, if there are more, the first three followed by et al; the title of journal articles or book chapters; the titles of journals abbreviated according to the style of Index Medicus; and the first and final page numbers of the article or chapter. Titles not in Index Medicus should be given in full. are:

Examples of common forms of references

1 International Committee of Medical Journal Editors. Uniform requirements for manuscripts submitted to Uniform requirements for manuscripts

2 Soter NA, Wasserman SI, Austen KF. Cold urticaria: release into the circulation of histmaine and eosinophil chemotactic factor of anaphylaxis during cold challenge. N Engl F Med 1976;294:687-90.

3 Weinstein L, Swartz MN. Pathogenic properties of invading micro-organisms. In: Sodeman WA Jr, Sodeman WA, eds. Pathologic physiology, mechanisms of disease. Philadelphia: W B Saunders, 1974:457-72. 